\title{
Die Reform des Risikoausgleichs als Vorbedingung für die Aufhebung des Kontrahierungszwangs?
}

\author{
S. Spycher
}

In einem Krankenversicherungssystem mit vorgegebenem Grundleistungspaket, Versicherungsobligatorium, Vorschrift zur Pro-Kopf-Einheitsprämie in jeder Versicherung und Wettbewerb zwischen den Versicherern ist die Etablierung eines Risikoausgleichs zwischen den Versicherern zwingend notwendig, damit der Wettbewerb funktionieren kann. Der Schweizerische Risikoausgleich mit den Faktoren Alter, Geschlecht und Region genügt dabei den Anforderungen an einen guten Risikoausgleich nicht. In der Folge konzentrieren sich die Versicherer auf die Selektion gesunder Versicherter statt auf das Management der Gesundheitskosten. Der Wettbewerb zwischen den Versicherern erfüllt die Erwartungen nicht. Eine Reform des Risikoausgleichs ist daher notwendig und kann aufgrund der vorhandenen Untersuchungen auch umgesetzt werden. Die geplante Aufhebung des Kontrahierungszwangs im ambulanten Bereich schafft für die Versicherer eine neue Möglichkeit der Risikoselektion, indem Ärztinnen und Ärzten mit kränkeren Patienten/-innen ein Vertragsabschluss verweigert werden kann. Der Kontrahierungszwang kann daher nur dann aufgehoben werden, wenn der Risikoausgleich stark verbessert wird.

\footnotetext{
a Dies erstaunt nicht, gibt es doch heute Versicherer, die pro Jahr bis zu 35 Prozent ihrer Prämieneinnahmen in den Risikoausgleich einzahlen [2].
}

Interessenbindungen

Sowohl der Autor wie auch das private Auftragsforschungsbüro BASS sind politisch vollständig unabhängig. Verschiedene Grundlagenarbeiten zum Risikoausgleich wurden im Auftrag des Bundesamtes für Sozialversicherung (heute: Bundesamt für Gesundheit) erarbeitet.

Korrespondenz:

Dr. rer. pol. Stefan Spycher

Büro für arbeits- und sozialpolitische

Studien BASS

Konsumstrasse 20

$\mathrm{CH}-3007$ Bern

Tel. 0313806080

Fax 0313983363

E-Mail:

stefan.spycher@buerobass.ch Markt auf. Sie konnten tiefe Prämien anbieten und zogen damit vor allem junge und gesunde Versicherte der herkömmlichen Krankenversicherer an. Die Mobilität der Versicherten war damals eingeschränkt, weil die Versicherer die Prämien nach dem Eintrittsalter in die Versicherung abstufen konnten (und bis 1993 auch nach Geschlecht). Daher kam es kaum zu Versicherungswechseln von älteren und meist kränkeren Personen. In der Folge bezahlten die jungen und gesunden Versicherten zunehmend tiefe Prämien, die älteren und meist kränkeren Personen entsprechend höhere Prämien. Die Solidarität zwischen «Gesund» und «Krank» wurde als gefährdet betrachtet [1]. Der Risikoausgleich greift hier korrigierend ein und teilt Geld zwischen den
Versicherern um: Diejenigen Kassen, die überdurchschnittlich viele ältere Versicherte bzw. überdurchschnittlich viele Frauen haben, erhalten Geld aus einem zentralen Fonds, die Versicherer, die in der umgekehrten Situation sind, zahlen Geld in den Fonds ein (Nullsummenspiel).

Der Risikoausgleich ist seit seiner Einführung

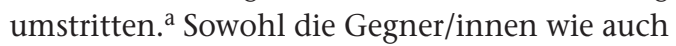
die Befürworter/innen des Risikoausgleichs argumentieren wettbewerbspolitisch: Die Gegner/innen sehen im Risikoausgleich eine ungerechtfertigte Subventionierung schlecht wirtschaftender Kassen (Strukturerhaltung), die Befürworter/innen eine berechtigte Beseitigung von Nachteilen im Wettbewerb, weil die Versicherer je nach Zusammensetzung ihres Versichertenkollektivs in einem Einheitsprämiensystem sehr unterschiedliche Ausgangslagen haben.

In der 2. Teilrevision des Krankenversicherungsgesetzes (KVG) ist keine wesentliche Reform des Risikoausgleichs geplant [3]. Hingegen soll der Kontrahierungszwang zwischen ambulanten Leistungserbringern/-innen und Versicherern aufgehoben werden [4]. Die Aufhebung des Kontrahierungszwangs steht in einem sehr engen Bezug zum Risikoausgleich, weil er die Risikoselektionsmöglichkeiten der Versicherer erhöht. traten vermehrt neue Krankenversicherer im

\section{Der Stand der Forschung zum Risiko- ausgleich}

\section{Entwicklung der Forschung}

Die Forschung zum Risikoausgleich begann in den frühen 1980er Jahren in den USA. Mit dem Aufkommen von Managed-Care-Organisationen (MCO) stellte sich die Frage, wie eine im voraus an die MCO zu bezahlende Pro-Kopf-Prämie (capitation) zu berechnen sei, damit die MCO die richtigen Anreize für eine qualitativ gute und zugleich kostengünstige Versorgung haben. Im staatlich finanzierten Medicare-Programm wurde eine Formel verankert, die vor allem auf den 
Variablen Alter, Geschlecht, Region und der Zugehörigkeit zur Sozialhilfe beruhte. Bald realisierte man, dass die MCO dadurch Anreize hatten, innerhalb von bestimmten Alters-Geschlechts-Gruppen die guten Risiken zu selektionieren und die kränkeren Personen zu «meiden». Es resultierte eine intensive Auseinandersetzung mit den Nachteilen der verwendeten Formel [5, 6]. Die Reformvorschläge zielten darauf ab, als Ergänzung zu Alter und Geschlecht einen adäquaten Morbiditätsindikator zu verwenden. Dazu wurden verschiedene Patientenklassifikationssysteme vorgeschlagen [7-10].

In Europa wurde die Diskussion zuerst in den Niederlanden aufgenommen [11]. Hier zeigte sich das Problem des Risikoausgleichs etwas anders als in den USA. Die Niederlande haben, ähnlich wie die Schweiz, ein Krankenversicherungssystem mit Wettbewerb zwischen Krankenversicherern. Die Steuerzahler/innen zahlen Beiträge in einen zentralen Fonds und nur weitgehend unbedeutende Kopfprämien direkt an die Versicherer. Nun stellt sich die Frage, welchen risikoadjustierten Beitrag die Versicherer pro Kopf ihrer Versicherten aus dem zentralen Fonds erhalten. Ausgehend von den Niederlanden dehnte sich die Forschung rasch auf Länder aus, die ebenfalls Krankenversicherer kennen, die in einem Wettbewerb zueinander stehen. ${ }^{\mathrm{b}}$

1995 erschien in der Schweiz die erste wissenschaftliche Arbeit zum Risikoausgleich, in der - wie im Ausland - darauf hingewiesen wurde, dass ein Risikoausgleich mit Alter und Geschlecht nicht ausreichend funktionieren kann [14]. In der Folge gab es zahlreiche Arbeiten, die sich zunehmend auch mit der Frage auseinandersetzten, wie der Risikoausgleich in der Schweiz angepasst werden könnte [15-18]. In Deutschland folgte der Einstieg in die Diskussion etwas verspätet, aber mit ähnlichen Ergebnissen wie in der Schweiz [19]. ${ }^{c}$

\section{Ergebnisse der Forschung}

Einleitend wurde erwähnt, dass der Bundesrat den Risikoausgleich in der Schweiz mit Blick auf die Tendenz zur Entsolidarisierung eingeführt hat. In der wissenschaftlichen Diskussion wird eigentlich nie mit der Solidarität zwischen «Gesund» und «Krank», sondern ausschliesslich wettbewerbspolitisch argumentiert. Ausgegangen wird von einem Krankenversicherungssystem, das ein Obligatorium, ein klar definiertes Grundleistungspaket und Versicherer, die in einem Wettbewerb zueinander stehen, kennt. Würde die Prämienbildung nicht reguliert, so würden sich in einem solchen System risikogerechte Prämien ergeben. Kränkere Menschen würden höhere Prämien bezahlen als gesündere, weil sie grössere Kostenrisiken für die Versicherer darstellen. Da in vielen Gesellschaften zwischen «Gesund» und «Krank» eine gewisse Solidarität gewünscht wird, greift der Staat mit Vorschriften in die Prämienbildung ein. Im Extremfall schreibt er vor, dass pro Kopf und unabhängig vom Gesundheitszustand dieselbe Prämie in jeder Versicherung zu verlangen sei. In der Schweiz dürfen die Prämien nach Regionen und drei Altersgruppen (Kinder, junge Erwachsene, Erwachsene) differenziert werden.

Die staatliche Vorschrift der Pro-Kopf-Einheitsprämie innerhalb einer Versicherung führt dazu, dass die Versicherer starke Anreize für die Risikoselektion haben. Je gesünder ihr Kollektiv ist, desto tiefer sind die Einheitsprämien und desto attraktiver ist der Versicherer im Markt. Aus gesundheitsökonomischer Sicht muss daher der eine staatliche Eingriff - die Vorschrift zur Einheitsprämie - mit einem weiteren staatlichen Eingriff zur Reduktion der Risikoselektionsanreize der Versicherer begleitet werden: Ein zwischen den Versicherern gut funktionierender Risikoausgleich muss eingeführt werden. Dieser verhindert, dass sich die Versicherer primär mit der Frage beschäftigen, wie sie sogenannte gute Risiken anziehen können, also gesunde (vornehmlich junge) Klienten/-innen, die kaum Kosten verursachen. Sind diese Risikoselektionsanreize nicht mehr vorhanden, können sich die Versicherer nur durch Anstrengungen in zwei Bereichen gegenüber den Konkurrenten auszeichnen: Zum einen müssen sie gute Dienstleistungen erbringen, so dass die Versicherten zufrieden sind. Zum anderen sollen sie mit dem Management der Gesundheitskosten dafür sorgen, dass sie günstiger sind als die Konkurrenz. Kostenvorteile können somit nicht mehr über die Risikoselektion, sondern nur noch über das bewusste Management der Kosten erreicht werden. Kurzum: Die Versicherer verwenden ihre Energie darauf, gute Versicherer zu sein, und nicht darauf, gesunde und somit günstige Versicherte anzuziehen. Es gibt somit konzeptionell ein «Entweder-Oder»: Entweder man lässt risikogerechte Prämien zu oder man hat Einheitsprämien mit einem Risikoausgleich, der die Risikoselektionsanreize beseitigt [20, 21].

Ein Risikoausgleich kann also die Risikoselektionsanreize der Versicherer beseitigen. Dazu muss er allerdings jene Kriterien berücksichtigen, die den Gesundheitszustand der Versicherten am genauesten erklärt. Mit den Kriterien Alter, Geschlecht und Region, die heute in der Schweiz zur Anwendung kommen, geschieht dies nur unzureichend. Damit werden nur etwa 
20 Prozent der erklärbaren Unterschiede im Gesundheitszustand der Versicherten abgedeckt [15]. Es lohnt sich für die Versicherer also nach wie vor, sich vor allem darauf zu konzentrieren, sogenannte gute Risiken anzuziehen [22].

\section{Ausgestaltung eines wirksamen Risiko- ausgleichs}

Der Risikoausgleich soll die Morbidität der Versicherten abbilden. Dazu wurden verschiedene Systeme von Diagnosekostengruppen entwickelt. Derartige Systeme gehen von den IC-Diagnosen aus und fassen sie derart zusammen, dass Gruppen mit ähnlichen Kostenrisiken für die Zukunft entstehen. Üblicherweise unterscheidet man dabei zwischen 15 und 30 Gruppen [20]. Es existieren Gruppierungen, die nur stationäre, nur ambulante oder stationäre und ambulante Diagnosen gemeinsam berücksichtigen. Was ist aber zu tun, wenn Diagnoseangaben nicht oder in mangelhafter Qualität zur Verfügung stehen? In der Literatur wurden dazu zwei Wege vorgeschlagen.

Zum einen wird darauf hingewiesen, dass andere Indikatoren als Diagnosen für den Gesundheitszustand beigezogen werden können. Genannt werden in diesem Zusammenhang etwa Indikatoren der Inanspruchnahme medizinischer Leistungen in den Vorjahren (z. B. Medikamente, Hospitalisierungen usw.) bzw. Indikatoren der Kosten der Inanspruchnahme in den Vorjahren (z. B. Vorjahreskostengruppen) [16].

Zum anderen wird vorgeschlagen, ergänzend zum Risikoausgleich einen Pool für Versicherte mit grossen gesundheitlichen Risiken und folglich sehr hohen Kosten einzuführen. Die Idee der Pools wurde in den 1990er Jahren in den Niederlanden entwickelt, weil damals der Risikoausgleich nicht über die Faktoren Alter und Geschlecht hinaus verändert werden konnte [23, 24]. Ein Pool würde wie folgt funktionieren: Die Versicherer können einen Teil der Kosten der teuersten Versicherten in einen Pool abgeben, der von allen Versicherern zusammen (Pro-KopfPrämie) oder über Steuern finanziert wird [15, 16]. Versicherer mit überdurchschnittlich vielen teuren Versicherten würden so netto entlastet, Versicherer mit einer unterdurchschnittlichen Häufigkeit der teuersten Versicherten zusätzlich belastet. Es würde sich ein zusätzlicher Risikoausgleich ergeben.

Das Abweichen von Diagnosekostengruppen hin $z u$ einfacheren Indikatoren des Gesundheitszustandes reduziert die Wirksamkeit des Risikoausgleichs zweifellos. Solange der Risikoausgleich jedoch in der Lage ist, die Selektionsanreize soweit zu reduzieren, dass die Versiche- rer nicht mehr bereit sind, viel Energie und Geld in die Selektion zu investieren, erfüllt er seinen Zweck.

\section{Reformvorschläge für die Schweiz}

Verschiedene Autoren beschäftigten sich in den letzten Jahren damit, eine Ergänzung der Risikoausgleichsformel zu finden [15, 18, 25]. Dabei zeigte sich zunehmend ein Konsens in dreierlei Hinsicht:

1. Die Formel sollte mit einem guten Indikator für den Gesundheitszustand ergänzt werden. Im Vordergrund stehen hierfür Diagnosekostengruppen, wie sie jüngst in einem Projekt des Schweizerischen Nationalfonds entwickelt worden sind [25].

2. Sind Diagnosekostengruppen nicht verfügbar, so besteht die Möglichkeit, einfachere und etwas weniger präzise Indikatoren für den Gesundheitszustand der Versicherten $\mathrm{zu}$ verwenden. $\mathrm{Zu}$ denken ist dabei an Vorjahreshospitalisationsgruppen oder an Vorjahreskostengruppen. Denkbar wären auch Medikamentenkostengruppen.

3. Wenn Diagnosekostengruppen nicht verfügbar sind, dann wäre weiter auch daran zu denken, einen Pool für Grossrisiken einzuführen. Die jüngsten Erfahrungen aus Deutschland zeigen, dass bei der Ausgestaltung darauf geachtet werden muss, dass die Kostenschwelle nicht zu hoch angesetzt wird und dass der Selbstbehalt der Versicherer nicht zu gross wird [26]. Zudem muss daran erinnert werden, dass ein Pool stets eine Second-best-Lösung ist und keinen Ersatz für einen gut ausgestalteten Risikoausgleich darstellt.

\section{Die Entwicklungen im Ausland}

Ähnliche Diskussionen wie in der Schweiz wurden auch in anderen Ländern geführt, die ein Gesundheitssystem haben, das sich auch auf den Wettbewerb zwischen den Versicherern abstützt. Allerdings haben sie - ausser in Israel - Reformen ausgelöst: In den USA wurden im Jahr 2001 im Medicare-Programm stationäre Diagnosekostengruppen eingeführt. In den Niederlanden hat man in den vergangenen Jahren Erfahrungen mit verschiedensten Instrumenten gemacht, unter anderem mit Medikamentenkostengruppen und mit einem Pool. Seit 2004 hat man nun ebenfalls stationäre Diagnosekostengruppen eingeführt. Auch in Deutschland wurde 2003 ein Pool implementiert. Auf das Jahr 2007 wurde die Einführung von Diagnosekostengruppen be- 
schlossen. In Belgien plant man die Implementierung von Diagnosekostengruppen auf das Jahr $2005 / 2006$. Ausser in Israel ist man in allen genannten Ländern über die Ausgleichsfaktoren Alter und Geschlecht hinausgegangen, weil erkannt wurde, dass mit Alter und Geschlecht alleine die Risikoselektionsanreize nicht beseitigt werden können.

\section{Politische Standpunkte in der Diskussion um den Risikoausgleich}

Im Rahmen der gesetzlich geforderten Wirkungsanalyse des KVG wurden zwei Studien zum Risikoausgleich erarbeitet, die die bekannten Ergebnisse der Forschung bestätigten und eine entsprechende Reform des Risikoausgleichs empfahlen [22, 27]. Der Bundesrat trat im Rahmen des ersten Anlaufs zur 2. Teilrevision des KVG nicht auf eine Reform ein und empfahl lediglich, den bis Ende 2005 befristeten Risikoausgleich unbefristet zu verankern [28]. Begründet wurde dieses Vorgehen vor allem damit, dass eine Risikoausgleichsreform das politische Revisionspaket überladen würde und ein Scheitern der ganzen Teilrevision bedeuten könnte. In der Bundesverwaltung wurden aber auch Befürchtungen laut, dass eine Ergänzung des Risikoausgleichs zu einem «Kosten»-Ausgleich führen würde, der letztlich ein erster Schritt Richtung Einheitskasse wäre. Der neuste Vorschlag des Bundesrates sieht nun eine Verlängerung des unveränderten Risikoausgleichs um 5 Jahre vor [3]. Es wird nicht deutlich, ob diese Verlängerung dazu dienen soll, den Risikoausgleich in dieser Zeit zu verbessern, oder ob er nach Ablauf der 5 Jahre abgeschafft werden soll.

Der Verband der Krankenversicherer (santésuisse) nahm zur Frage des Risikoausgleichs im Unterschied $\mathrm{zu}$ einzelnen Versicherern nicht eindeutig Stellung: Die Versicherer, die in den Ausgleich einzahlen, möchten ihn so wie heute belassen oder sogar abschaffen. Die Versicherer, die Geld aus dem Ausgleich erhalten, möchten ihn erweitern.

Im Rahmen der Diskussionen zur 1. sowie zur 2. Teilrevision wurden im Parlament verschiedentlich Vorstösse zur Verbesserung des Risikoausgleichs eingereicht. Verantwortlich dafür waren vor allem die Nationalräte Gross (SP) und Rychen (SVP) [15]. Ebenfalls wurde von der SP ein Vorstoss zur Einführung eines Pools lanciert. Alle Vorstösse wurden jedoch abgelehnt.

\section{Der Risikoausgleich und der Kontrahie- rungszwang}

Im Rahmen der 2. Teilrevision des KVG wird vorgeschlagen, den Kontrahierungszwang zwischen den ambulanten Leistungserbringern/-innen und den Versicherern aufzuheben [4]. Die Aufhebung würde den Versicherern ein neues Instrument für die Risikoselektion in die Hände geben: Sie könnten mit dem gezielten Abschluss von Verträgen erreichen, dass sie kränkere Patienten/-innen an andere Versicherer abgeben können. Wenn z. B. eine Ärztin dafür bekannt wäre, dass sie viele HIV-Patienten/-innen versorgt, würde die Vertragsverweigerung evtl. dazu führen, dass die Versicherten dieser Ärztin zu demjenigen Versicherer wechseln, der mit der besagten Ärztin noch einen Vertrag abschliesst.

Die Aufhebung des Kontrahierungszwang schafft also eine neue Möglichkeit der Risikoselektion. Ob diese Möglichkeit von den Versicherern genutzt wird, hängt von verschiedenen Faktoren ab. Zum einen sicher davon, ob die Versicherer mit negativen Konsequenzen rechnen müssen, z.B. mit der Abwanderung von (gesunden) Versicherten, die ein solches Verhalten nicht akzeptieren. Zum anderen spielt die Ausgestaltung des Risikoausgleichs eine zentrale Rolle. Wenn der Risikoausgleich so ausgestaltet ist, dass die Versicherer geringe Risikoselektionsanreize haben, dann werden sie von dieser neuen Selektionsmöglichkeit keinen Gebrauch machen. Mit anderen Worten: Eine Reform des heutigen Risikoausgleichs, die die Anreize der Versicherer so weit als möglich reduziert, Energie und Geld in die Suche nach gesunden Klienten/-innen zu investieren, ist eine unabdingbare Voraussetzung für die Aufhebung des Kontrahierungszwangs [29].

Gesetzt den Fall, dass Diagnosekostengruppen in die Ausgleichsformel eingeführt würden, kann man davon ausgehen, dass die Anreize nicht weiter reduziert werden können. Allerdings gibt es eine wichtige Relativierung zu erwähnen. Die in der Schweiz vorgeschlagenen Diagnosekostengruppen basieren auf stationären Diagnosen. Im ambulanten Bereich - und um den geht es bei der Aufhebung des Kontrahierungszwangs - werden heute noch keine Diagnosen systematisch erfasst. Daher können auch keine Diagnosekostengruppen im ambulanten Bereich gebildet werden. Dies führt dazu, dass der Risikoausgleich eine Lücke aufweisen wird: Die Versicherten, die hohe ambulante, aber keine stationären Kosten aufweisen (ambulante Kosten über 10000 Franken pro Jahr), werden nur mangelhaft berücksichtigt. Hier eröffnen 
sich Selektionsanreize für die Versicherer, die zum bewussten Auswählen von Ärztinnen und Ärzten führen könnten. Wie häufig dies in der Praxis der Fall ist, muss mangels entsprechender Untersuchungen offenbleiben. Mit den Daten, die über TARMED gesammelt werden, eröffnen sich neue Möglichkeiten, Indikatoren für die ambulante Praxis zu bilden. Diese könnten u.U. die genannte Lücke schliessen.

\section{Diskussion}

Die Ergebnisse der Forschung sind eindeutig: In einem System mit Pro-Kopf-Einheitsprämien innerhalb der Kassen und einem Wettbewerb zwischen den Krankenversicherern braucht es einen Risikoausgleich zwischen den Kassen, der die Risikoselektionsanreize beseitigt. Der Risikoausgleich muss so ausgestaltet sein, dass er die Differenzen im Gesundheitszustand der Versicherten berücksichtigt. Am besten eignen sich dafür Diagnosekostengruppen, die Versicherte mit ähnlichen Kostenrisiken zusammenfassen. Wird der Risikoausgleich nicht korrekt ausgestaltet, konzentrieren sich die Versicherer weiterhin auf die Selektion gesunder Versicherter und kümmern sich nur unzureichend um die Qualität der Versorgung und um das Management der Gesundheitskosten. Beides lässt sich in der Schweiz momentan beobachten, weil der Risikoausgleich nur die Faktoren Alter, Geschlecht und Region berücksichtigt. Eine Reform des Risikoausgleichs ist daher, auch ohne anderweitige Änderungen des KVG, dringend notwendig.

Nun gewinnt die Situation durch die geplante Aufhebung des Kontrahierungszwangs zwischen den Versicherern und den ambulanten Leistungserbringern/-innen an Brisanz. Die Aufhebung gibt den Versicherern ein zusätzliches Selektionsinstrument in die Hände. Dieses werden sie nur dann nicht benutzen, wenn es sich nicht mehr lohnt, in die Suche nach guten Risiken (gesunde, kostengünstige Versicherte) zu investieren. Dafür sorgt der Risikoausgleich jedoch nur, wenn er richtig ausgestaltet ist. Der Kontrahierungszwang kann unmöglich aufgehoben werden, wenn der Risikoausgleich nicht gleichzeitig verbessert wird. Ansonsten würden kränkere Versicherte Opfer der neuen Selektionsmöglichkeiten.

Es stehen heute verschiedene Möglichkeiten zur Verfügung, den Risikoausgleich anzupassen. Allerdings kann aufgrund der vorliegenden Ergebnisse nicht abschliessend beurteilt werden, ob die Ergänzung der Formel mit stationären Diagnosekostengruppen - der besten der zur Ver- fügung stehenden Möglichkeiten - ausreichen wird, um vollständig zu verhindern, dass die Versicherer die neuen Selektionsmöglichkeiten wahrnehmen. Mittelfristig muss auf jeden Fall daran gedacht werden, auch ambulante Diagnosen bzw. Indikatoren für die ambulante Praxis mit zu berücksichtigen.

\section{Literatur}

1 Bundesrat. Botschaft über die Revision der Krankenversicherung vom 6. November 1991.

2 Bundesamt für Sozialversicherung. Aufsichtsdaten OKP gemäss Art. 31 KVV. 2003. www.sozialversicherungen.admin.ch/?lng=de.

3 Bundesrat. Botschaft betreffend die Teilrevision des Bundesgesetzes über die Krankenversicherung (Strategie und dringliche Punkte), 26. Mai 2004. www.bag.admin.ch/kv/projekte/d/Botschaft \%201A\%20d\%20mit\%20Artikeln_250504.pdf.

4 Bundesrat. Botschaft betreffend die Teilrevision des Bundesgesetzes über die Krankenversicherung (Vertragsfreiheit), 26. Mai 2004. www. bag.admin.ch/kv/projekte/d/Botschaft $\% 201 \mathrm{~B}$ \%20d\%20mit\%20Artikeln_250504.pdf.

5 Newhouse JP, Willard G, Manning E, Keeler B, Sloss EM. Adjusting capitation rates using objective health measures and prior utilization. Health Care Financing Review 1989;3:41-54.

6 Giacomini M, Luft HS, Robinson JC. Risk adjusting community rated health plan premiums: a survey of risk assessment literature and policy applications. Ann Rev Public Health 1995; 16:401-30.

7 Greenwald LM et al. Risk adjustment for the Medicare program: lessons learned from research and demonstrations. Inquiry 1998;35(2):193-209.

8 Ash A, Porell F, Gruenberg L, Sawitz E, Beiser A. Adjusting Medicare capitation payments using prior hospitalization data. Health Care Financing Rev 1989;10(4):17-29.

9 Ellis RP, Pope GC, Iezzoni LI, Ayanian JZ, Bates DW, Burstin H, Ash AS. Diagnosis-based risk adjustment for Medicare capitation payments. Health Care Financing Rev 1996;17(3):101-28.

10 Weiner JP, Dobson A, Maxwell SL, Coleman K, Starfield BH, Anderson GF. Risk-adjusted Medicare capitation rates using ambulatory and inpatient diagnosis. Health Care Financing Rev 1996; 17(3):77-99.

11 Van de Ven W, van Vliet R. How can we prevent cream skimming in a competitive health insurance market? In: Zweifel P, Frech III HE (eds.). Health Economics Worldwide. Amsterdam: Kluver Acadmic Publisher; 1992. p. 23-46.

12 Van de Ven WPMM, Beck K, Buchner F, Chernichovsky D, Gardiol L, Holly A, et al. Risk adjustment and risk selection on the sickness fund insurance market in five European countries. Health Policy 2003;65(1):75-98.

13 Beck K (Hrsg.). Reformstau beim Risikoausgleich? - Internationale Erfahrungen und konkrete Lösungen für die Schweiz. Luzern: Eigenverlag; 2004. 
14 Beck K, Debever Hilfiker A, Kocher G, Schocker E, Bardenhofer D. Die Solidarität im Wandel. Schriftenreihe der Schweizerischen Gesellschaft für Gesundheitspolitik (SGGP), 1995, Band 48.

15 Spycher S. Risikoausgleich in der Krankenversicherung - Notwendigkeit, Ausgestaltung und Wirkungen. Bern: Paul Haupt Verlag; 2002.

16 Spycher S. Risikoausgleich und Poollösungen («Grossrisikopool») in der obligatorischen Krankenversicherung. Im Auftrag des Bundesamtes für Sozialversicherung, Bern. Beiträge zur sozialen Sicherheit, Forschungsbericht Nr. 19/2003, EDMZ, Bern.

17 Beck K, Spycher S, Holly A, Gardiol L. Risk adjustment in Switzerland. Health Policy 2003; 65(1):63-74.

18 Beck K. Risiko Krankenversicherung - Risikomanagement in einem regulierten Krankenversicherungsmarkt. Bern (erscheint demnächst).

19 Jacobs K, Cassel D, Wasem J. Zur Wirkung des Risikostrukturausgleichs in der gesetzlichen Krankenversicherung. Eine Untersuchung im Auftrag des Bundesministeriums für Gesundheit. Endbericht vom 15. Februar 2001.

20 Van de Ven WPMM, Ellis RP. Risk adjustment in competitive health plan markets. In: Newhouse JP, Culyer AJ. Handbook of Health Economics. Amsterdam: Elsevier; 2000. p. 757-845.

21 Zweifel P, Breuer M. Risikoausgleich und Finanzierung der Krankenversicherung: Feststellung und Perspektiven. Bericht zuhanden der Cosama. 2001.

22 Spycher S. Wirkungsanalyse des Risikoausgleichs in der Krankenversicherung. Beiträge zur sozialen Sicherheit, Forschungsbericht Nr. 1/1999, EDMZ, Bern.
23 Van Barneveld EM, Van Vliet RCJA, Van de Ven WPMM. Mandatory high-risk pooling: an approach to reducing incentives for cream skimming. Inquiry 1996;33(2):133-43.

24 Van Barneveld EM, Lamers LM, Van Vliet RCJA, Van de Ven WPMM. Mandatory pooling as a supplement to risk-adjusted capitation. Social Science and Medicine 1998;47(2):223-32.

25 Holly A, Gardiol L, Eggli Y, Yalcin T. Health-Based Risk-Adjustment in Switzerland: An Exploration Using Medical Infomation from Prior Hospitalization. Final report. Research financed by the Swiss National Fund. 2003.

26 Wasem J. Deutscher Risikostrukturausgleich: Laufende Optimierung der Formel. Folien zum Vortrag am Meeting des Risk-Adjustment-Network in Luzern, 5. Februar 2004.

27 Spycher S. Reform des Risikoausgleichs in der Krankenversicherung? Studie 2: Empirische Prüfung von Vorschlägen zur Optimierung der heutigen Ausgestaltung. Beiträge zur sozialen Sicherheit, Forschungsbericht Nr. 3/2000, EDMZ, Bern.

28 Bundesrat. Botschaft betreffend die Änderung des Bundesgesetzes über die Krankenversicherung vom 18. September 2000, Bundesblatt, 741-823.

29 Spycher S. Der Risikoausgleich im Rahmen der Teilrevision des Krankenversicherungsgesetzes. Definitiver Schlussbericht der vom Bundesamt für Sozialversicherung eingesetzten Arbeitsgruppe «Risikoausgleich». 2004. www.buerobass.ch/ neu_aktuell_d.html. 